\title{
Positron Age-Momentum-Correlation (AMOC) Measurements on Organic Liquids
}

\author{
Petra Castellaz, Andreas Siegle, ${ }^{\dagger}$ and Hermann Stoll* \\ Max-Planck-Institut für Metallforschung \\ Heisenbergstr.1, D-70569 Stuttgart, Germany
}

Received: November 18, 2002

\begin{abstract}
By means of correlated measurements of the individual lifetimes of positrons (called positron ages) and of the Doppler shifts of their $2 \gamma$-annihilation radiation (Age-Momentum Correlation, acronym AMOC) the room-temperature behaviour of positrons and positronium in n-hexane, tetramethylsilane, benzene, methanol, ethanol, $n$ butanol, n-hexanol, n-octanol, and water has been investigated. The annihilation parameters of "free" positrons and of para- and orthopositronium (lifetimes and Doppler-broadened lines) have been deduced from a two-dimensional analysis of the AMOC data. The lifetimes of positrons are found to be related to the electronic polarizability of the investigated liquid. The behaviour of the Doppler-broadened free-positron and orthopositronium linewidths is explained in terms of structural and dipolar properties of the liquid molecules.
\end{abstract}

\section{Introduction}

Positrons $\left(\mathrm{e}^{+}\right)$, the positively charged anti-particles of the electrons $\left(\mathrm{e}^{-}\right)$, are widely used as non-destructive mobile probes in condensed matter, e.g., for the study of vacancy-like defects in crystals. In many isolating materials positronium $\left(\mathrm{Ps}=\mathrm{e}^{+} \mathrm{e}^{-}\right.$), the bound state of a positron and an electron, is at our disposal as an additional, electrically neutral probe. With some justification, Ps can be regarded as the lightest isotope of hydrogen. It may undergo chemical reactions analogous to those of hydrogen (e.g., oxidation or complex formation). In contrast to most other branches of reaction chemistry, in which information on the reactions is primarily derived from studying educts and products, positronium chemistry offers the possibility to observe the behaviour of one of the reaction partners, $v i z$. the positronium, rather directly on time scales between $10^{-11}$ and $10^{-8} \mathrm{~s}$.

In the past, positronium chemists studied primarily liquid solutions that were based on a variety of organic and inorganic solvents. ${ }^{1-3}$ This was particularly true of one of the key subjects of positronium chemistry, viz. the formation of positronium in liquids. Here, experiments on scavenger solutions provided insight into the processes involved. ${ }^{4}$ More recently, this "chemical" approach, based on investigating $\mathrm{e}^{+}$ and Ps reactions with different reactants, has been supplemented by comparing Monte Carlo simulations of the processes envisaged in Mogensen's spur model of Ps formation ${ }^{3,5}$ with positron annihilation and electron-radiolysis experiments. ${ }^{6,7}$

Clearly, reliable knowledge of the behaviour of $\mathrm{e}^{+}$and Ps in the pure solvents is a prerequisite for a deeper understanding of $\mathrm{e}^{+}$and Ps reactions in solutions. The present work investigates the behaviour of $\mathrm{e}^{+}$and $\mathrm{Ps}$ in various pure organic and inorganic liquids in a "physico-chemical" approach, in which material parameters such as surface tension, polarizability, or electron mobility are systematically varied. It is based on the AMOC (= Age Momentum Correlation) technique, which has already proved to be an excellent tool for studying $\mathrm{e}^{+}$and Ps reactions in liquid solutions..$^{8-10}$

Section 2 of this publication reviews the experimental evidences for positron states occurring in organic liquids. The

*Corresponding author. E-mail: stoll@mf.mpg.de. FAX: +49711-1932.

†Present address: Robert Bosch GmbH, D-70442 Stuttgart,
AMOC set-up used for the measurements and the sample preparation are briefly described in Section 3. The experimental results will be presented in Section 4 and discussed in detail in Section 5, followed by conclusions in Section 6.

\section{Basic Processes}

The conventional techniques for studying positron annihilation provide information either on the spectrum of positron lifetimes or on the distribution function of the momenta of annihilating positron-electron pairs. The positron lifetime spectrum is deduced from the distribution function of the positron ages, i.e., the times spent by the positrons in the sample before they annihilate with electrons. The reciprocal positron lifetimes associated with the distinct positron states, called annihilation rates, are proportional to the electron densities at the sites at which the positrons annihilate. The information on the momentum distribution of the annihilating $\mathrm{e}^{+} \mathrm{e}^{-}$ pairs is obtained from the $2 \gamma$-annihilation radiation. In the rest system of an annihilating $\mathrm{e}^{+} \mathrm{e}^{-}$pair the two $\gamma$-quanta are emitted collinearly, each of them with an energy of $m_{\mathrm{e}} c^{2}=511 \mathrm{keV}$, where $m_{\mathrm{e}}$ is the electron mass and $c$ the velocity of light in vacuum. In the laboratory system, the $\mathrm{e}^{+} \mathrm{e}^{-}$pairs in general have finite momenta. The momentum components parallel to the emission axis of the annihilation photons, the so-called longitudinal momenta, give rise to Doppler shifts of the photon energies. For an ensemble of positrons annihilating from a certain positron state these shifts result in a Doppler-broadened annihilation line characteristic for this distinct positron state. The AMOC technique, to be described in detail in Section 3.1, combines lifetime and Doppler-broadening spectrometry by correlated measurements of the positron age and the Doppler shift of one of the annihilation quanta. Therefore, it allows us not only to separate the momentum distribution associated with the annihilation processes with the different lifetimes from each other but also to follow the behaviour of the $\mathrm{e}^{+}$as a function of the positron age.

In most Ps-forming liquids, at least three distinct positron states may be detected, viz. (i) the singlet Ps ground state, called parapositronium (p-Ps), in which the $\mathrm{e}^{+}$annihilate with their "own" electrons, (ii) free positrons $\left(\mathrm{e}^{+}\right)$, annihilating predominantly with valence electrons of the surrounding molecules, and (iii) orthopositronium (o-Ps), the triplet Ps ground state. The self-annihilation of isolated o-Ps would lead to the Germany. 
emission of three $\gamma$-quanta with the total energy $2 m_{\mathrm{e}} c^{2}$. In condensed matter, however, it is much more probable that the $\mathrm{e}^{+}$of o-Ps annihilates with an electron with opposite spin of one of the surrounding molecules. This so-called pick-off process results in the emission of two $511 \mathrm{keV}$ photons.

In the case of "intrinsic" annihilation of p-Ps (= annihilation of the positrons with its "own" electrons) the Doppler broadening provides information on the residual kinetic energy of $p$ Ps. In the case of annihilation of "free positrons" or "pick-off" annihilation of the positrons of o-Ps with "foreign" electrons the Doppler broadening reflects the momentum distribution of the annihilating electrons since the momenta of the electrons involved are generally higher compared with those of the positrons.

The characteristic annihilation features of the three positron states described above are summarized in Table 1. The very weak Doppler broadening associated with the annihilation of thermalized p-Ps is due to the fact that here both annihilation partners have very low momenta. Non-thermalized p-Ps,

TABLE 1: Annihilation Characteristics of the Positrons in Ps-Forming Materials

\begin{tabular}{llrl}
\hline State & Annihilation mode & \multicolumn{1}{c}{ Lifetime } & Doppler broadening \\
\hline p-Ps & self-annihilation & $\approx 0.12 \mathrm{~ns}$ & very weak \\
free $^{+}$ & with valence $\mathrm{e}^{-}$ & $0.4-0.5 \mathrm{~ns}$ & strong \\
o-Ps & pick-off & $0.5-5 \mathrm{~ns}$ & strong \\
o-Ps & $3 \gamma$-annihilation & $140 \mathrm{~ns}$ & strong, asymmetrical \\
\hline
\end{tabular}

however, may give rise to appreciable Doppler broadening of the $2 \gamma$-annihilation line. ${ }^{11,12}$

However, Doppler-broadening measurements do not show p-Ps annihilation lines as narrow as they ought to be. This is due to the limited resolution of the energy detectors used in the experiment. It leads to a convolution of the annihilation lineshape with the energy resolution function of the detector. In order to achieve the "true" annihilation linewidths it is necessary to deconvolute the measured annihilation lines after the measurement.

Lifetime measurements are very suitable for determining the lifetime of o-Ps and the intensity of the o-Ps state, which is the relative number of positrons annihilating from this state, since in time regions above $1 \mathrm{~ns}$, o-Ps is the only surviving species, due to its long lifetime. Therefore, a "pure" o-Ps lifetime spectrum is observed in this time region. However, the limited resolution of the lifetime spectrometers often makes the separation of the p-Ps and free $\mathrm{e}^{+}$states difficult, since they annihi-

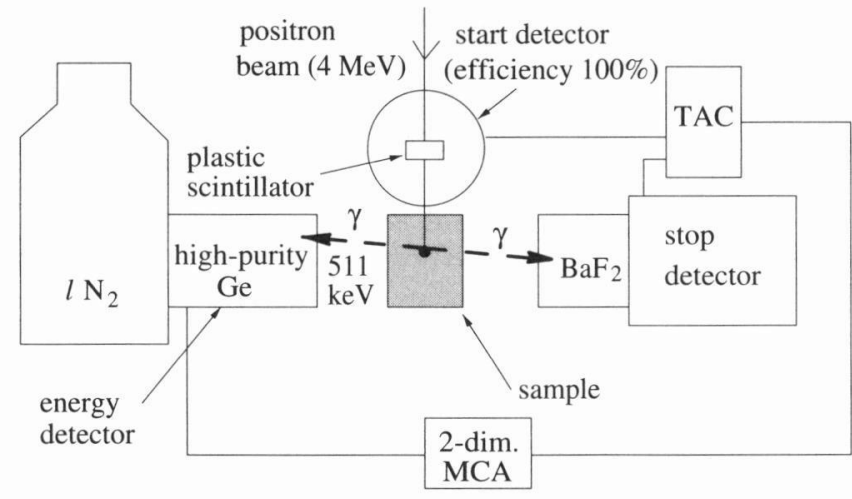

Figure 1. Schematic of the AMOC set-up installed at the Stuttgart $\mathrm{MeV}$ positron beam. The start signal for the positron-age measurement is generated by the passage of a positron through a plastic scintillator, the stop signal by the detection of an annihilation photon in a $\mathrm{BaF}_{2}$ scintillator. The second annihilation photon is used for the measurement of the Doppler shift by means of a liquid-nitrogen cooled high-purity-germanium detector. late predominantly in the same time region ( 0 to $0.6 \mathrm{~ns})$. The AMOC method overcomes this difficulty by allowing us to determine the Doppler-broadened lineshapes and the annihilation rates of the various positron states rather accurately. ${ }^{14,15}$ In contrast to age-integrated Doppler-broadening measurements, AMOC measures the Doppler-broadening associated with the various positron states in their characteristic lifetime ranges. For positron-chemistry studies of positronium-forming materials such as organic liquids AMOC is the ideal tool since it combines the o-Ps-sensitive lifetime measurement with the Doppler measurement, which permits easy identification of $\mathrm{p}$ Ps with its characteristic narrow momentum distribution.

\section{Experimental}

3.1. AMOC Spectrometer and Visualization. Figure 1 is a schematic of the AMOC set-up installed at the Stuttgart MeV positron beam. ${ }^{16-18}$ Positrons from a $4 \mathrm{MeV}$ beam passing through a plastic scintillator with a high detection efficiency generate the start signal for the age measurement with a timeto-amplitude converter (TAC) before being implanted into the sample. The stop signal is derived from the detection of one of the annihilation quanta by a $\mathrm{BaF}_{2}$ scintillator. The second annihilation quantum is used for the correlated measurement of the Doppler shift by means of a high-purity Germanium detector installed opposite to the stop detector. The signals from the age and the energy measurement are correlated by a two-parameter multi-channel analyser (MCA). For the data presented here the time resolution was about 250 ps (FWHM), the energy resolution (monitored by a simultaneous measurement of the ${ }^{103} \mathrm{Ru} \gamma$-photo line) about $1.4 \mathrm{keV}$ (FWHM), and the count rate typically about 500 triple coincidences per second.

By means of a two-parameter multichannel analyser the annihilation events are arranged in a two-dimensional histogram. This histogram may be visualized by an AMOC relief (Figure 2) where the number of counts is plotted logarithmically versus the positron age and the energy of the annihilation radiation. Summing all counts along the age axis results in the time-integrated Doppler-broadened lineshape of the photon energy, summing along the energy axis in the usual lifetime spectrum.

For the visualization of the correlation between Doppler broadening and positron age it is instructive to compute the

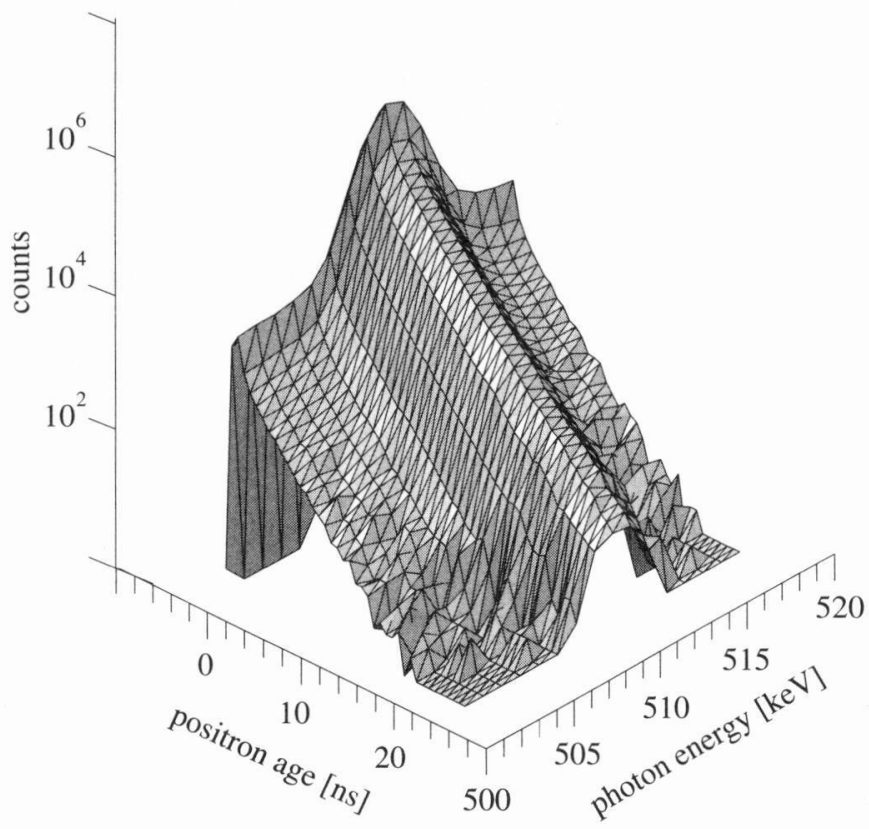

Figure 2. Room temperature AMOC relief of benzene. The counts are plotted vs. the individual positron lifetime (= positron age) and the energy of the annihilation quanta. 


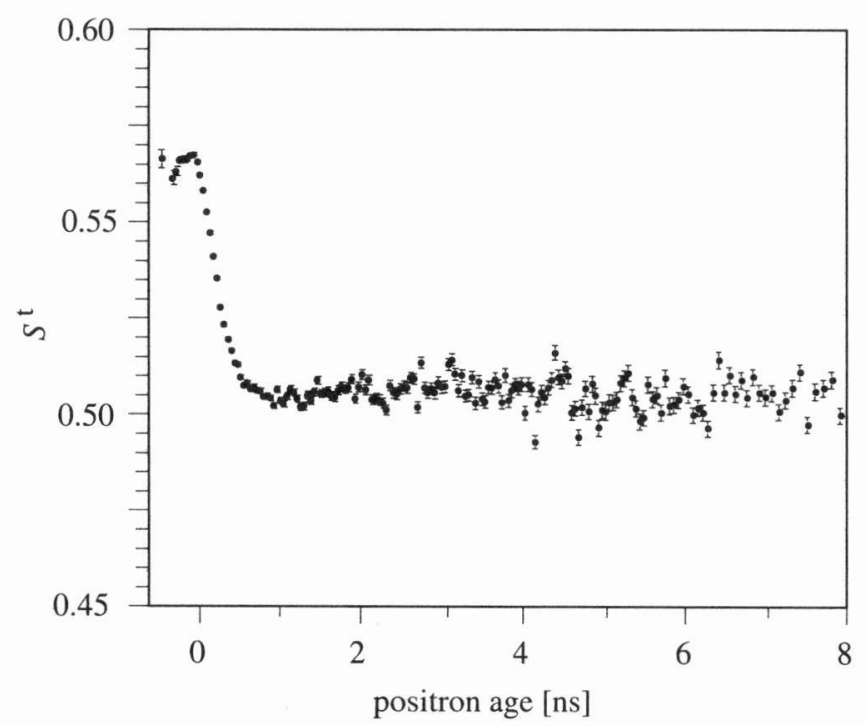

Figure 3. Lineshape function $S^{t}(t)$ of benzene at room temperature, calculated from the AMOC relief shown in Figure 2.

age-dependent lineshape function $S^{t}(t)$ (Figure 3) by determining separately for each time channel of the AMOC relief (see Figure 2) the lineshape parameter $S$ (defined as the ratio of the number of counts in the centre of the annihilation line to the number of counts in the entire line ${ }^{19}$ ) and plotting it versus the positron age. A large $S$ parameter corresponds to a narrow Doppler-broadening of the annihilation line, a small one to a broad line.*

The characteristic lineshape function $S^{t}(t)$ of a material forming positronium (see Figure 3 ) shows large $S^{\mathrm{t}}(t)$ values at short positron ages, resulting from the self-annihilation of thermalized or nearly thermalized p-Ps. This is followed by a steep decrease of $S^{t}(t)$. At larger $t$ the level corresponding to the rather broad momentum distribution of the electrons which annihilate with the positrons of o-Ps via the pick-off process is reached (see also Table 1). In cases where the momentum distribution of the electrons annihilating with free positrons is different from the momentum distribution of the electrons annihilating with the positrons of o-Ps via a pick-off process, the annihilation of the free positrons shows up as a change in $S^{t}(t)$ in a time range around 1 ns (see, e.g., Figure 7). Clearly, this is not the case with benzene (cf. Figure 3).

3.2. Sample Preparation. AMOC measurements were performed at room temperature on benzene $\left(\mathrm{C}_{6} \mathrm{H}_{6}\right)$, n-hexane $\left(\mathrm{C}_{6} \mathrm{H}_{14}\right)$, tetramethylsilane $\left(\left(\mathrm{CH}_{3}\right)_{4} \mathrm{Si}\right.$, abbreviated TMS $)$, methanol $\left(\mathrm{CH}_{3} \mathrm{OH}\right)$, ethanol $\left(\mathrm{C}_{2} \mathrm{H}_{5} \mathrm{OH}\right)$, n-butanol $\left(\mathrm{C}_{4} \mathrm{H}_{9} \mathrm{OH}\right)$, n-hexanol $\left(\mathrm{C}_{6} \mathrm{H}_{13} \mathrm{OH}\right)$, n-octanol $\left(\mathrm{C}_{8} \mathrm{H}_{17} \mathrm{OH}\right)$, and water $\left(\mathrm{H}_{2} \mathrm{O}\right)$. All samples were degassed by the freeze-pump-thaw method ${ }^{20}$ and filled into special glass vessels with thin windows that allowed implantation of the $\mathrm{MeV}$ positrons into the samples.

\section{Results}

The AMOC reliefs were analysed using a two-dimensional fitting procedure. ${ }^{21}$ In order to make full use of the measured AMOC data a two-dimensional model function representing the number of counts as a function of positron age and energy of the annihilation quanta is fitted to the AMOC relief without prior data reduction. On the age axis, each positron state, i.e. free positrons, p-Ps and o-Ps, is represented by an exponential decay function convoluted with the time resolution function of the spectrometer. On the energy axis, one Doppler-broadened Gaussian lineshape corresponds to each positron state. The annihilation with core electrons is taken into account by a broad Gaussian line of low amplitude.

For all liquids investigated, three positron states (p-Ps, $\mathrm{e}^{+}$, and o-Ps) sufficed for describing the data adequately. There was no need to take reactions among these positron states into consideration. In particular, no evidence of a fourth positron state in n-hexane was found as proposed earlier in the literature. ${ }^{3,22}$ Moreover, in contrast to Reference 23, no significant deviation from the statistical ratio $1: 3$ of the formation probabilities of p-Ps and o-Ps was observed; hence, it suffices to report the fraction $I_{\mathrm{Ps}}$ of positrons annihilating from positronium states, the so-called positronium yield. The fraction of positrons not forming Ps is thus given by $1-I_{\mathrm{Ps}}$.

In all Ps-forming materials examined so far by the AMOC technique, juvenile broadening arising from the delayed slowing-down of positronium ${ }^{11,12,18}$ was observed: in contrast to the charged positrons, the electrically neutral positronium which is formed at an early stage of the slowing-down process cannot loose its kinetic energy via ionization of molecules or creation of excitons but only in a less effective way via phonon excitation. Therefore, a considerable amount of positronium is not thermalized when it undergoes annihilation. Its kinetic energy results in a large Doppler broadening visible at young positron ages. This juvenile broadening was taken into account by a two-state rate-equation model. ${ }^{12}$ This model approximates the Ps energy loss by allowing for transitions between epithermal and slowed-down Ps. An improved analysis of AMOC data on Ps slowing-down is suggested by Seeger. ${ }^{13}$

The annihilation parameters of the three positron states obtained by the two-dimensional analysis program for the liquids examined in the present work are listed in Table 2 together with the typical statistical errors.

TABLE 2: Annihilation Parameters as Obtained from the Two-Dimensional Data Analysis. The last line of the table gives the typical statistical errors for the various quantities. Since all data analyses were based on similar statistics, the statistical errors are about the same for all samples. The Gaussian linewidths (standard deviations) $\sigma_{i}$ of the annihilation lines are given after deconvolution with the energy resolution function.

\begin{tabular}{|c|c|c|c|c|c|c|c|}
\hline Sample & $\tau_{\mathrm{p}-\mathrm{Ps}}[\mathrm{ps}]$ & $\tau_{\mathrm{e}+}[\mathrm{ps}]$ & $\tau_{\mathrm{o}-\mathrm{Ps}}[\mathrm{ps}]$ & $I_{\mathrm{Ps}}$ & $\sigma_{\mathrm{p}-\mathrm{Ps}}[\mathrm{keV}]$ & $\sigma_{\mathrm{e}+}[\mathrm{keV}]$ & $\sigma_{\mathrm{o}-\mathrm{Ps}}[\mathrm{keV}]$ \\
\hline benzene & 118 & 414 & 3059 & 0.563 & 0.33 & 0.89 & 0.89 \\
\hline n-hexane & 127 & 452 & 3724 & 0.466 & 0.27 & 0.92 & 0.89 \\
\hline TMS & 134 & 506 & 4619 & 0.698 & 0.22 & 0.87 & 0.84 \\
\hline methanol & 139 & 442 & 2997 & 0.293 & 0.26 & 1.05 & 0.96 \\
\hline ethanol & 132 & 435 & 3584 & 0.294 & 0.27 & 1.03 & 0.92 \\
\hline n-butanol & 126 & 420 & 3323 & 0.311 & 0.27 & 1.04 & 0.93 \\
\hline n-hexanol & 123 & 417 & 3166 & 0.318 & 0.27 & 1.03 & 0.93 \\
\hline n-octanol & 122 & 412 & 3087 & 0.330 & 0.28 & 1.03 & 0.93 \\
\hline water & 131 & 400 & 1848 & 0.365 & 0.43 & 1.09 & 1.07 \\
\hline stat.error & \pm 2 & \pm 1 & \pm 3 & \pm 0.005 & \pm 0.02 & \pm 0.01 & \pm 0.01 \\
\hline
\end{tabular}

*The absolute value of $S$ has no immediate physical significance since the width of the centre region of the annihilation lines is deliberately chosen to give $S$ parameters near 0.5 in order to achieve maximum sensitivity against small variations of the linewidth. 


\section{Discussion}

5.1. Orthopositronium Lifetime. It is generally accepted that the pick-off lifetime of the o-Ps state in liquids can be described by the bubble model proposed by Ferrell. ${ }^{24}$ The Ps bubble arises from the "quantum" pressure of the Ps motion which is in equilibrium with the surface tension of the surrounding liquid. $\mathrm{TaO}^{25}$ showed that the bubble model yields the following semi-empirical relation between the pick-off annihilation rate $\lambda_{0-P s}$ and the macroscopic surface tension $\gamma$ :

$$
\lambda_{\mathrm{o}-\mathrm{Ps}}=1.9 \times 10^{-9}\left(\mathrm{~s}^{-1} \mathrm{~m}^{1 / 2} \mathrm{~N}^{-1 / 2}\right) \times \gamma^{1 / 2} .
$$

This relation was shown to be valid for a large variety of organic and inorganic liquids.

Figure 4 shows the o-Ps annihilation rate as a function of the surface tension for various liquids. The good agreement of the results from the present AMOC measurements with eq 1 (solid line in Figure 4), which was derived earlier, ${ }^{20,25}$ is obvious. More recent publications discuss modifications of the bubble model concerning a modified surface tension of the Ps bubble ${ }^{26}$ (cf. Section 5.3) and a more elegant calculation of the bubble energy. ${ }^{27}$

5.2. Free-Positron Lifetime. In 1968 , Gray et al. ${ }^{20}$ showed that "partial" annihilation rates $\lambda_{\text {partial }}^{*}$ may be derived for different organic functional molecular groups and that the specific pick-off annihilation rate $\lambda_{\mathrm{po}}^{*}=\lambda_{\mathrm{po}} / V_{M}\left(\lambda_{\mathrm{po}}\right.$ denotes the pick-off annihilation rate and $V_{M}$ the molar volume) of an organic liquid may be determined by summing up these partial rates according to the given molecular structure:

$$
\lambda_{\mathrm{po}}^{*}=\Sigma \lambda_{\text {partial }}^{*}
$$

It was known that the electronic polarizability does also have this property. From this coincidence, Gray et al. derived a correlation between electronic polarizability and pick-off annihilation rate. It was $\mathrm{Tao}^{25}$ who pointed out that the pickoff annihilation rate may be related to any quantity which represents the electronic interactions between the molecules. Both surface tension $\gamma$ and polarizability are, in fact, such quantities; since it is not to be expected that the behaviour of electrically neutral positronium is influenced by the polarizability of the surrounding molecules the surface tension is surely better suited as a parameter of the bubble model.

However, a correlation between the polarizability of the medium and the annihilation rate would be more straightforward for the free positron since it is a charged particle. To our knowledge, this correlation has not been discussed in the literature yet. This may be due to the limited time resolution of the

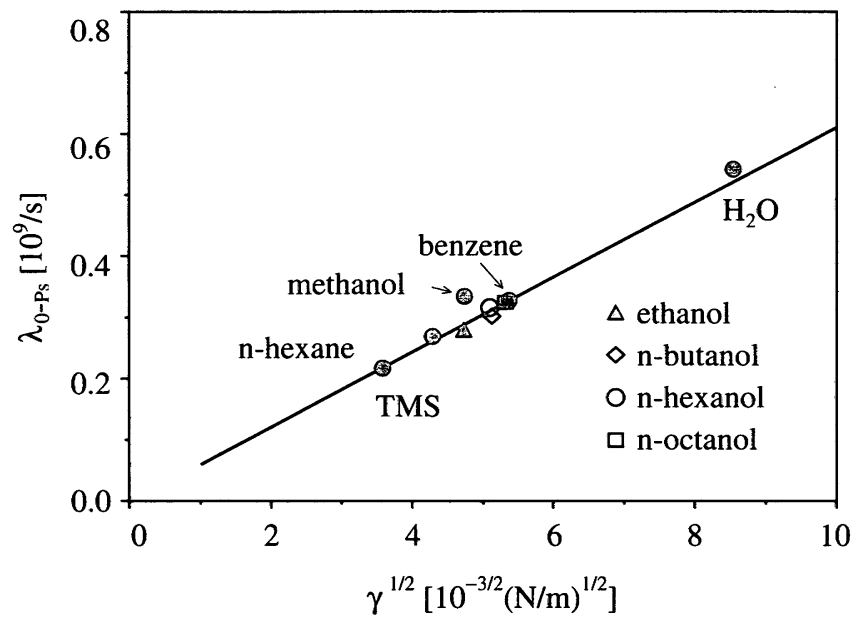

Figure 4. o-Ps pick-off annihilation rate $\lambda_{\mathrm{o}-\mathrm{Ps}}$ vs. surface tension $\gamma$ (Ref. 29) for benzene, n-hexane, tetramethylsilane (TMS), water, and various normal alcohols. The solid line was calculated from eq 1 . lifetime spectrometers which prevented an accurate determination of the free-positron lifetime in the presence of another short-lived component such as p-Ps.

A positron implanted into matter polarizes its surroundings due to its electric charge, attracting the electrons of the molecules. The larger the polarizability, the more the electron density increases at the positron site and the faster the positron annihilates. Figure 5 shows the specific annihilation rate of the free positrons $\lambda_{\mathrm{e}_{+}}{ }^{*}$ determined from AMOC measurements as a function of the polarizability $\alpha$ of the liquids determined from refraction measurements. ${ }^{28}$ As expected, the annihilation rate increases with increasing polarizability of the surrounding molecules. A fit of a straight line

$$
\lambda_{\mathrm{e}+}{ }^{*}(\alpha)-\lambda_{\mathrm{e}+}{ }^{*}(0)=b \times \alpha
$$

to the data points in Figure 5 leads to a proportionality factor $b=0.555 \times 10^{-3} \mathrm{JmC}^{-2} \mathrm{~s}^{-1}$ and a constant $\lambda_{\mathrm{e}+}{ }^{*}(0)=2.4 \times 10^{-20}$ $\mathrm{m}^{3} \mathrm{~s}^{-1}$.

5.3. Doppler Broadening of the Annihilation Lines. As already mentioned in Section 4, the linewidths of the Dopplerbroadened lines of the different positron states may be determined rather accurately by the AMOC method. The two-dimensional analysis uses Gaussian lines with standard deviations $\sigma_{i}$ for the modelling of the Doppler-broadened lineshapes for all occurring positron states $i$. If we assume that the true velocity distribution for the motion of positronium in the bubble is not far away from a Maxwell velocity distribution, this will lead to a Gaussian Doppler broadening of the annihilation line. ${ }^{17}$ From this assumption, the mean kinetic energy of p-Ps can be derived as

$$
E_{\mathrm{p}-\mathrm{Ps}}^{\mathrm{kin}}=3 \frac{\sigma_{\mathrm{p}-\mathrm{Ps}}^{2}}{m_{e} c^{2}}
$$

where $m_{\mathrm{e}}$ is the electron mass and $c$ the velocity of light. If we generalize this relation for the annihilation of free positrons and o-Ps, we have to consider that in both cases the momentum of the annihilating $\mathrm{e}^{+} \mathrm{e}^{-}$pair is mainly determined by the momentum of the electron (which generally is bound to a molecule) whereas the positron can be regarded as completely thermalized. As only one particle, the electron, contributes to the momentum distribution, we have to calculate its mean kinetic energy according to Reference 17

$$
E_{\mathrm{e}-}^{\mathrm{kin}}\left(\mathrm{e}^{+}\right)=6 \frac{\sigma_{\mathrm{e}+}^{2}}{m_{e} c^{2}} \text { and } E_{\mathrm{e}-}^{\mathrm{kin}}(\mathrm{o}-\mathrm{Ps})=6 \frac{\sigma_{\mathrm{o}-\mathrm{Ps}}^{2}}{m_{e} c^{2}}
$$

from the deconvoluted Gaussian linewidths $\sigma_{i}$ obtained from the two-dimensional data analysis.

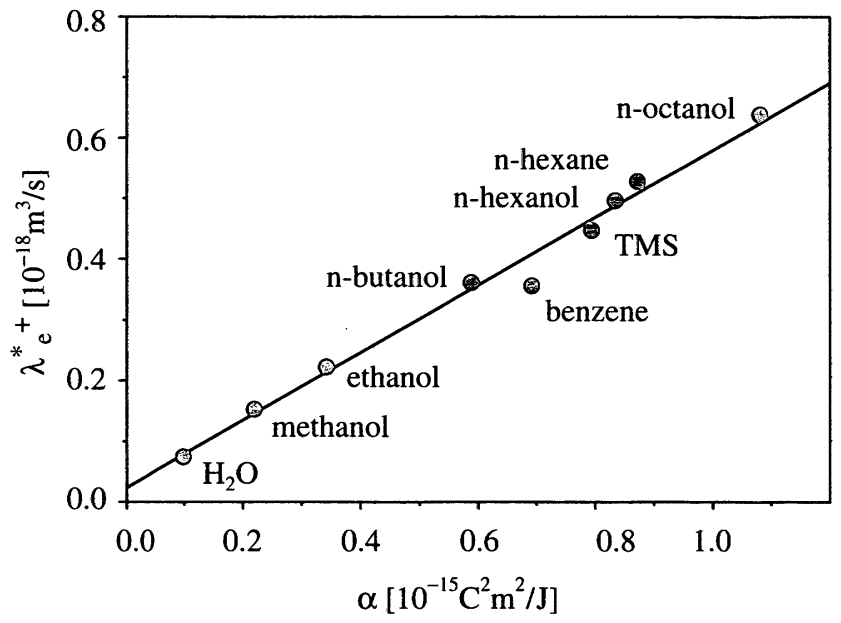

Figure 5. Specific free-positron annihilation rate $\lambda_{e_{+}}^{*}=\lambda_{e_{+}} / V_{M}$ vs. polarizability $\alpha$ (Ref. 28 ) for benzene, $n$-hexane, tetramethylsilane (TMS), water, and various normal alcohols. 
$E^{\mathrm{kin}}{ }_{\mathrm{p}-\mathrm{Ps}}$ was calculated from the Doppler-broadened linewidths of p-Ps given in Table 2, and the results were plotted versus the surface tension $\gamma$ of the measured liquids in Figure 6. The mean kinetic energy of p-Ps is well above the thermal energy of $k_{\mathrm{B}} \mathrm{T}=0.025 \mathrm{eV}$ at room temperature. It increases with increasing surface tension, i.e., with decreasing bubble radius, as predicted from the bubble model. ${ }^{25}$ This model can be used for a calculation of the Ps kinetic energy in the bubble using a simple square potential in which the Ps is caged. ${ }^{24}$ Under the assumption of infinitely high potential walls Mogensen ${ }^{3}$ gives

$$
\mathrm{E}_{\mathrm{Ps}_{\mathrm{S}}}^{\mathrm{kin}}=d \times \gamma^{1 / 2} \text {, with } d=7.68 \mathrm{eVN}^{-1 / 2} \mathrm{~m}^{1 / 2} .
$$

Equation 6 gives a qualitative description of our experimental results (cf. full lines in Figure 6) but the calculated energies are overestimated by about a factor of two. This is thought to be expected when a model with infinitely high potential walls is used. A comparison of our results with those from the refined bubble model of Byakov et al. ${ }^{26}$ shows a rather good agreement for the Ps kinetic energies in n-hexane, benzene, TMS, ethanol, octanol, and water.

In Figure 7 the lineshape functions $S^{\mathrm{t}}(t)$ obtained for $\mathrm{n}$ hexane and n-hexanol are compared. Both curves show the characteristic features of positronium formation. The lineshape functions at old positron ages, which can be attributed to the pick-off annihilation of o-Ps, are nearly the same. In contrast to the lineshape function of $n$-hexanol showing a pronounced minimum in the region of 0.5 to $2 \mathrm{~ns}, S^{\mathrm{t}}(t)$ of n-

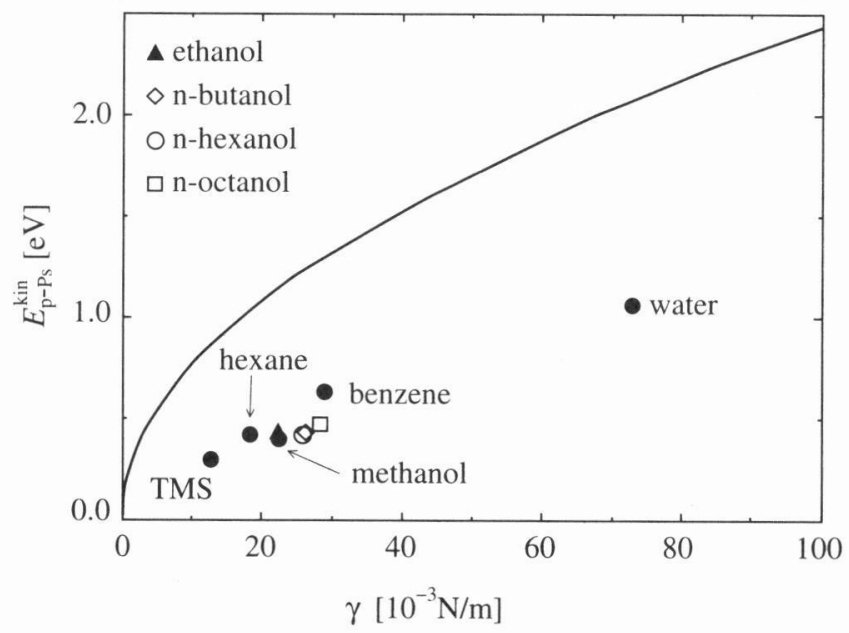

Figure 6. p-Ps mean kinetic energy $E^{\text {kin }}{ }_{\text {p.Ps }}$ vs. surface tension $\gamma$ (Ref. 29 ) for various liquids. The solid line was calculated from eq 6.

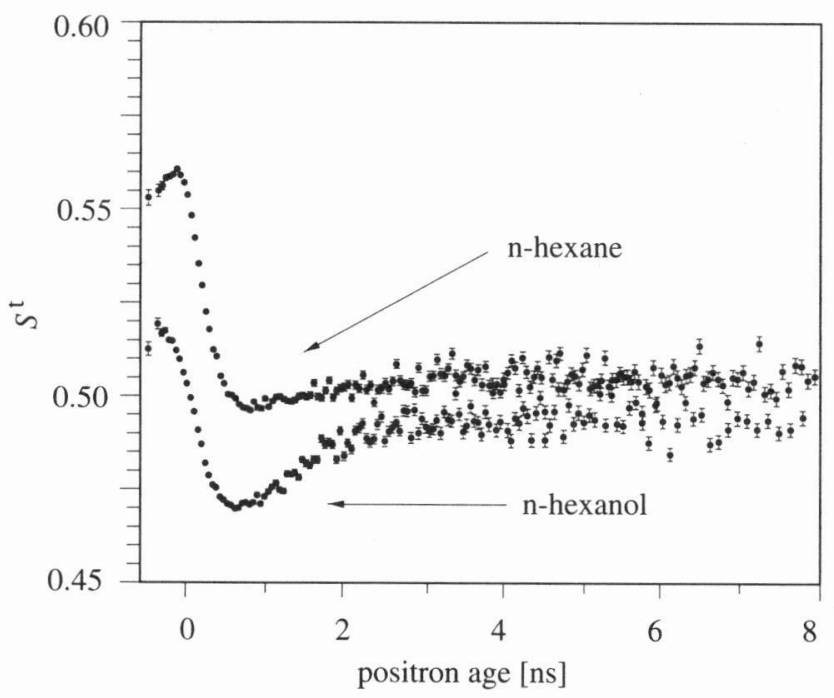

Figure 7. Lineshape functions $S^{\mathrm{t}}(t)$ obtained for $\mathrm{n}$-hexane and $\mathrm{n}$ hexanol calculated from the AMOC reliefs. hexane passes through a quite shallow minimum. Since the minima occur in the time range where the annihilation of the free positrons takes place, it is straightforward to suppose that the electrons which annihilate with the free positrons in nhexanol have a broader momentum distribution than those in $\mathrm{n}$-hexane. The fact that the lineshape function in $\mathrm{n}$-hexane shows only a shallow minimum seems to indicate that the free positrons and the positrons of o-Ps in n-hexane annihilate with electrons of essentially the same momentum distribution.

In order to test whether this finding may be generalized, the mean kinetic energies of the electrons annihilating with free positrons $E^{\mathrm{kin}}{ }_{\mathrm{e}-}\left(\mathrm{e}^{+}\right)$and those annihilating with the positrons of o-Ps $E_{\text {e- }}^{\text {kin }}$ (o-Ps) were calculated from eq 5 and are shown in Figure 8 for all liquids measured. The two energies are almost equal for the nonpolar liquids benzene, n-hexane, and TMS, but different by more than $3 \mathrm{eV}$ for the alcohols which are polar molecules owing to the large electronegativity of the oxygen of the hydroxyl group. In polar liquids, free positrons are expected to be solvated by the molecular dipoles. Only the water molecule, which has the largest dipole moment of all liquids examined, does not fit into this pattern. This exception will be discussed in Section 5.5. Figure 9 shows the schematic molecular structure of methanol with the permanent partial charges $\delta^{+}$and $\delta^{-}$.

Free positrons are attracted by the negative partial charge on the hydroxyl group resulting in a solvation sphere of solvent molecules around the positrons. They can, therefore, annihilate mainly with the p-electrons of the oxygen since these electrons are nearest. As shown schematically by the example of $\mathrm{OH}^{-}$in Figure 10, the non-binding p-electrons have a larger energy than the other electrons of the molecules and hence cause a larger Doppler broadening on annihilation. Positronium as a neutral particle does, in contrast to the free positron, not interact with the dipole moment of the molecules.

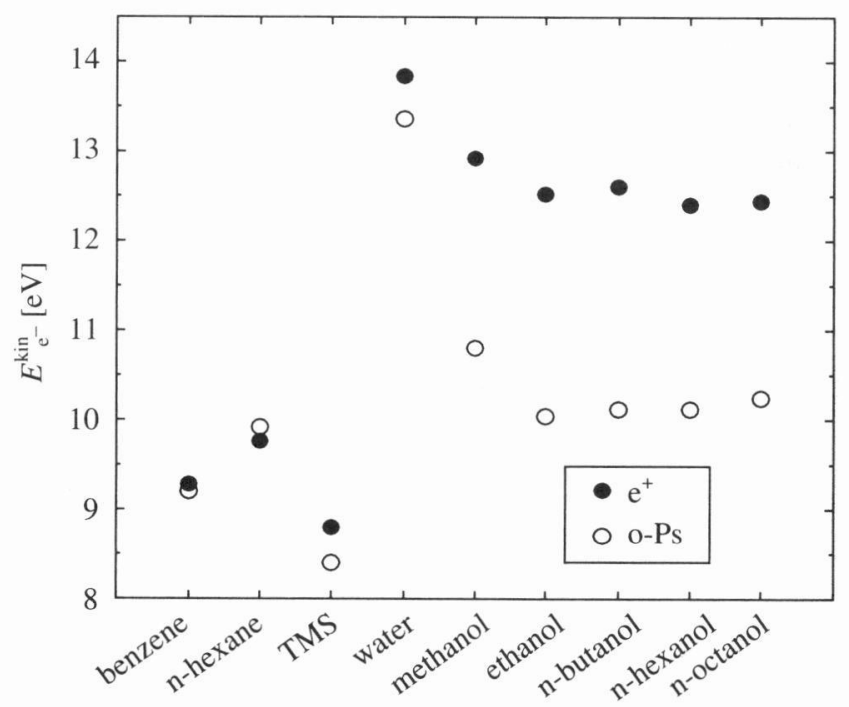

Figure 8. Mean kinetic energies $E^{\text {kin }}{ }_{\mathrm{e}^{-}}$of the electrons annihilating with free positrons ( $\mathrm{e}^{+}$, full symbols) and with o-Ps (open symbols) for some polar and nonpolar liquids.

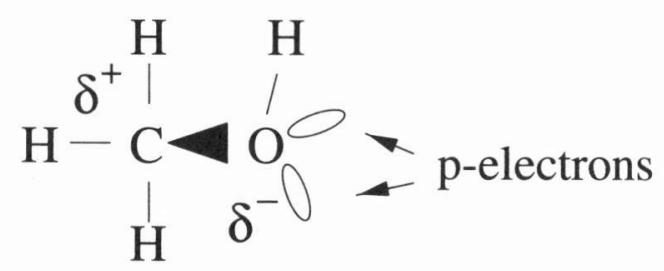

Figure 9. Molecular structure of methanol showing the partial charges $\delta^{+}$and $\delta^{-}$and the p-electrons (ellipses). The black triangle denotes the polarization of the binding electrons towards the electronegative oxygen atom. 


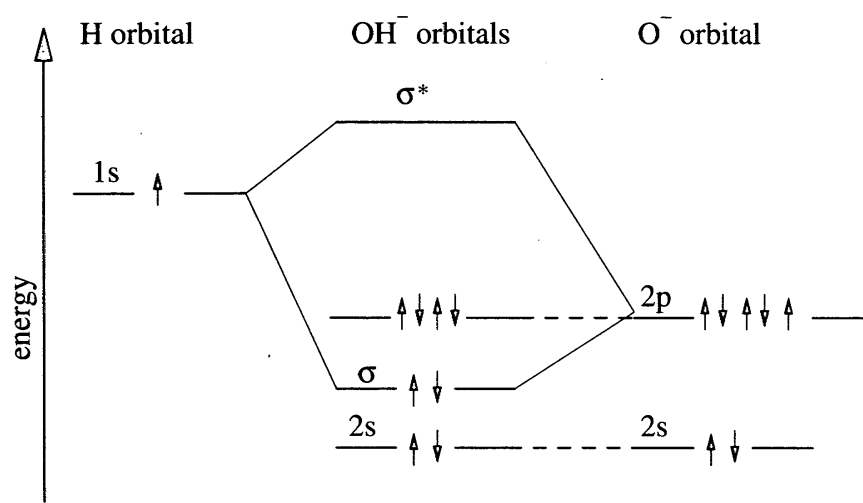

Figure 10. Schematic diagram for the energy levels of a heteropolar chemical bond on the example of $\mathrm{OH}^{-} . \sigma$ and $\sigma^{*}$ denote the binding and non-binding molecular orbitals, respectively.

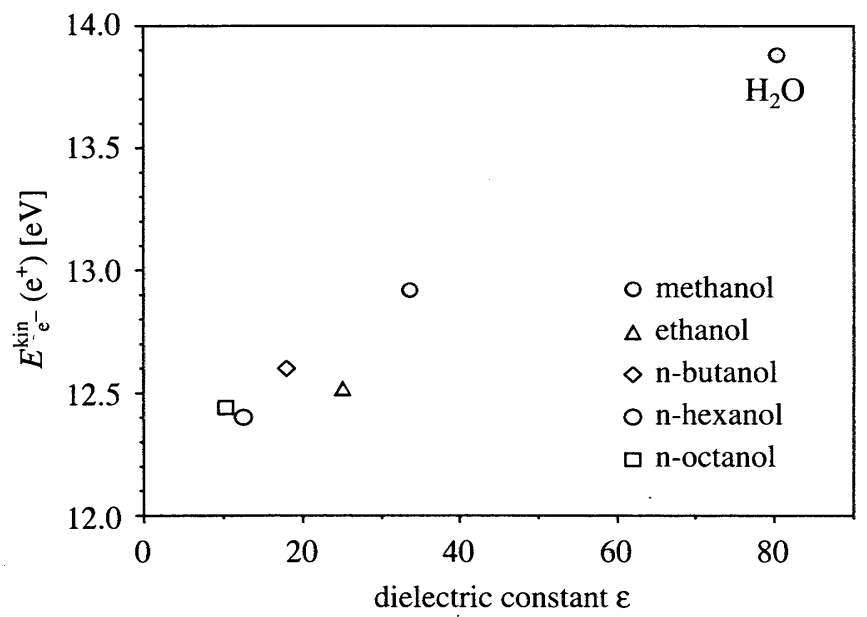

Figure 11. Mean kinetic energy $E^{\mathrm{kin}}{ }_{\mathrm{e}-}$ of the $\mathrm{e}^{+} \mathrm{e}^{-}$pair for freepositron ( $\left.\mathrm{e}^{+}\right)$annihilation vs. static dielectric constant $\varepsilon$ (Ref. 28) for $\mathrm{C}_{n} \mathrm{H}_{2 n+1} \mathrm{OH}(n=0,1,2,4,6$, and 8$)$.

The pick-off process is, therefore, not selective and its Doppler-broadening linewidth is a measure for the momentum distribution of all outer electrons of the molecules. In the case of non-polar molecules there is no dipole moment, free positrons are not solvated and, therefore, the free positrons and the o-Ps annihilate with the same type of electrons.

Our findings agree with the work of Byakov et al. ${ }^{30}$ who focus on the orientation of liquid molecules perpendicular to the Ps bubble surface: their experimental results suggest that the OH-group of methanol points away from the Ps bubble. The positron of o-Ps, therefore, annihilates predominantly with "low energy" electrons on the methyl group.

When mean kinetic energies are discussed we should not forget that o-Ps is also caged in the positronium bubble. This effect also contributes to the o-Ps linewidth. As shown above, the bubble model (eq 6) gives a rather good qualitative description for the kinetic energy of p-Ps in the bubble. The kinetic energy of o-Ps must be approximately the same. This cannot be tested by experiment, since o-Ps annihilates via pick-off process. As the momentum distribution of the bound electrons annihilating in the pick-off process is much broader than the momentum distribution due to the motion of the Ps in the bubble, it gives only a small contribution to the measured annihilation linewidth. It is, therefore, neglected in this discussion.

5.4. Positron Annihilation in Polar Liquids: Normal Alcohols. In order to test the influence of the strength of the molecular dipole, the results obtained on a series of normal alcohols with increasing carbon chain length, and, therefore, decreasing dipole moment can be compared. Figure 11 shows the mean kinetic energy of the electrons annihilating with free positrons plotted versus the static dielectric constant $\varepsilon$ of the

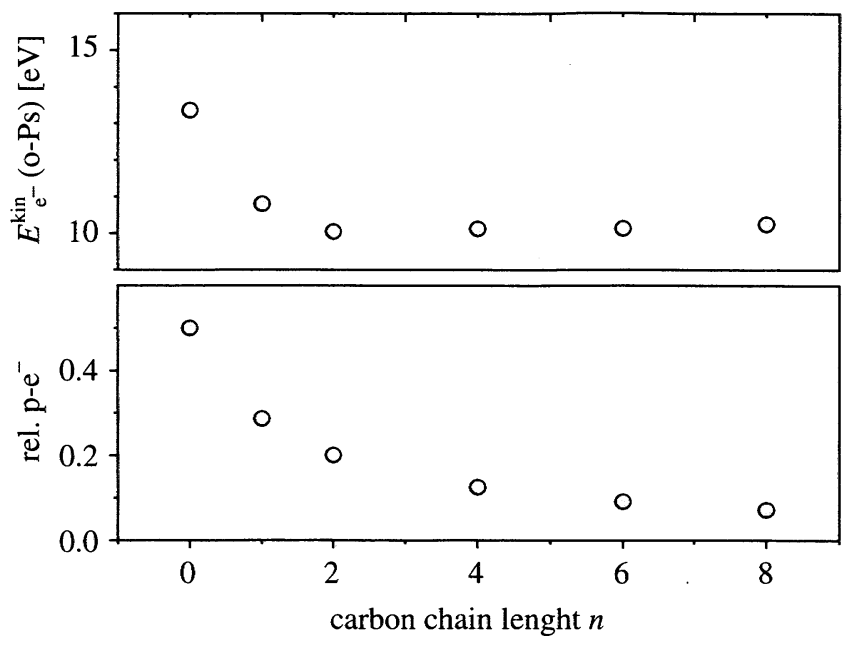

Figure 12. Upper part: Mean kinetic energy $* * \mathrm{E}^{\mathrm{kin}}{ }_{\mathrm{p}-\mathrm{Ps}}$ of the $\mathrm{e}^{+} \mathrm{e}^{-}$pairs for the annihilation of o-Ps vs. carbon chain length $n$ for $\mathrm{C}_{n} \mathrm{H}_{2 n+1} \mathrm{OH}(n$ $=0,1,2,4,6$, and 8). Lower part: Fraction of valence electrons being p-electrons as a function of $n$.

liquid. The energy $E^{\mathrm{kin}}{ }_{\mathrm{e}-}\left(\mathrm{e}^{+}\right)$increases with increasing $\varepsilon$ and is the highest in water which, from this point of view, may be regarded as an alcohol of chain length $n=0$. It may be concluded from this result that with increasing dipole moment the probability for the free positron to annihilate with one of the p-electrons at the oxygen is also rising.

Finally the seemingly exceptional behaviour of water has to be discussed where the mean kinetic energies of the electrons annihilating with free positrons $E_{\mathrm{e}-}^{\mathrm{kin}}\left(\mathrm{e}^{+}\right)$and with o-Ps $E^{\mathrm{kin}}{ }_{\mathrm{e}-}$ (o-Ps) are very high and almost equal as shown in Figure 8 . In the previous section we gave an explanation why the energy of electrons annihilating with free positrons is high, but what is the cause for the high energy in the pick-off process (upper part of Figure 12)? The lower part of Figure 12 gives a possible explanation for this finding. Since positronium is an electrically neutral particle there should be no preferences of its position respective to the molecular structure and its positron can annihilate via pick-off with the same probability with any valence electron of the molecules. In water, fifty per cent of the valence electrons are p-electrons. This number decreases with increasing carbon chain length, as shown in Figure 12. The more valence electrons are p-electrons the larger the Doppler broadening in the pick-off process.

\section{Conclusions}

The present AMOC measurements performed on benzene, n-hexane, tetramethylsilane, water, methanol, ethanol, nbutanol, $n$-hexanol, and n-octanol have shown that the annihilation parameters of free positrons and of para- and orthopositronium such as positron annihilation rates and Doppler-broadened linewidths are correlated with macroscopic and structural features of the liquids. The pick-off annihilation rate of o-Ps can be well described by the bubble model where the intramolecular electrical forces are expressed in terms of the surface tension of the liquid. A clear correlation between the electronic polarizability of the molecules and the lifetime of the free positrons could be established for the first time. The detailed information on the Doppler-broadened linewidth of the distinct positron states obtained by AMOC allowed us to demonstrate that in polar liquids free positrons are solvated and, therefore, annihilate with electrons with a momentum distribution which is different to that of the electrons responsible for pick-off annihilation.

The experiments performed so far indicate that the fraction of free positrons annihilating from a solvated state increases with increasing dipole moment. 
Acknowledgements. The authors would like to thank all of those who were involved in the Stuttgart MeV positron beam. This paper would not have been possible without the work of all of them. In particular we would like to thank Prof. Dr. A. Seeger for his continuous support and Dr. I. Billard, Strasbourg, for many fruitful discussions.

\section{References}

(1) J. H. Green and J. Lee, Positronium Chemistry (Academic Press, New York, 1964).

(2) V. I. Goldanskii and V. P. Shantarovich, in Modern Physics in Chemistry, edited by E. Fluck and V. I. Goldanskii (Acadmic Press, New York, 1974), p.269.

(3) O. E. Mogensen, Positron Annihilation in Chemistry, Springer Series in Physics Vol.58 (Springer, Berlin etc., 1995).

(4) G. Duplâtre, J. Ch. Abbé, A.G.Maddock, and A. Haessler, J. Chem. Phys. 72, 89 (1980).

(5) O. E. Mogensen, J. Chem. Phys. 60, 998 (1974).

(6) N. Gee and G. R. Freeman, J. Chem. Phys. 90, 5399 (1989).

(7) I. Billard, T. Goulet, J. -P. Jay-Gerin, and A. Bonnenfant, Mater. Science Forum 255-257, 104 (1997).

(8) H. Schneider, A. Seeger, H. Stoll, I. Billard, M .Koch, U. Lauff, and J. Major, Journal de Physique IV Coll. C4, 69 (1993).

(9) P. Castellaz, J. Major, C. Mujica, H. Schneider, A. Seeger, A. Siegle, H. Stoll, and I. Billard, J. Radioanal. Nucl. Chem. Art. 210, 457 (1996).

(10) P. Castellaz, Untersuchung von chemischen Reaktionen des Positrons und des Positroniums in Flüssigkeiten mit Hilfe der Lebensalter-Impuls-Korrelation (AMOC), Dr. rer. nat. Thesis, Universität Stuttgart, ISBN 3-8958-934-2 (Cuvillier, Göttingen, 1997).

(11) A. Seeger, Appl. Surf. Sci. 85, 8 (1995).

(12)H. Schneider, A. Seeger, A. Siegle, H. Stoll, P. Castellaz, and J. Major, Appl. Surf. Sci. 116, 145 (1997).

(13) A. Seeger, Radiation Physics and Chemistry 58, 411 (2000).
(14) A. Seeger, Mater. Science Forum 255-257, 1 (1997).

(15) H. Stoll, P. Castellaz, S. Koch, J. Major, H. Schneider, A. Seeger, and A. Siegle, Mater. Science Forum 255-257, 92 (1997).

(16) H. Stoll, M. Koch, K. Maier, and J. Major, Nucl. Inst. Meth. 56/57, 582 (1991).

(17) A. Siegle, Positronenzerstrahlung in kondensierter Materie-eine Untersuchung mit der Methode LebensalterImpuls-Korrelation (AMOC), Dr. rer. nat. Thesis, Universität Stuttgart, ISBN 3-89712-129-8 (Cuvillier, Göttingen, 1998).

(18) H. Stoll, MeV Positron Beams, in Positron Beams and Their Applications, edited by P. Coleman (World Scientific Singapore, 2000), p. 237.

(19)I. K. MacKenzie, J. A. Eady, and R. R. Gingerich, Phys. Lett. 33A, 279 (1970).

(20) P. R. Gray, C. F. Cook, and G. P. Sturm, J. Chem. Phys. 48, 1145 (1968).

(21) A. Siegle, H. Stoll, P. Castellaz, J. Major, H. Schneider, and A. Seeger, Appl. Surf. Sci. 116, 140 (1997).

(22) Sz. Vass, Cs. Szeles, K. Süvegh, and A. Vértes, Mat. Sci. Forum 105-110, 1749 (1992).

(23) O. E. Mogensen and F. M. Jacobsen, Chem. Phys. 73, 223 (1982).

(24) R. A. Ferrell, Rev. Mod. Phys. 28, 308 (1956).

(25) S. J. Tao, J. Chem. Phys. 56, 5499 (1972).

(26) V. M. Byakov and S. V. Stepanov, Rad. Phys. Chem. 58, 87 (2000).

(27)T. Mukherjee, B. N. Ganguly, and B. Dutta-Roy, J. Chem. Phys. 107, 467 (1997).

(28) H. Stuart and Th. Gast in Landolt-Börnstein: Zahlenwerte und Funktionen Vol.II/6, edited by J. Bartels, P. Ten Bruggencate, H. Hansen, K. H. Hellwege, K. Schäfer, and E. Schmidt (Springer, Berlin etc., 1959).

(29)E. Beger in Landolt-Börnstein: Zahlenwerte und Funktionen Vol.II/3, edited by J. Bartels, P. Ten Bruggencate, H. Hansen, K. H. Hellwege, K. Schäfer, and E. Schmidt (Springer, Berlin etc., 1959).

(30) V. M. Byakov and S. V. Stepanov, Mater. Science Forum 255-257, 284 (1997). 